\title{
Energy Minimizing Vehicle Routing Problem
}

\author{
İmdat Kara ${ }^{1}$, Bahar Y. Kara ${ }^{2}$, and M. Kadri Yetis ${ }^{3}$ \\ ${ }^{1}$ Başkent University, Dept. Ind. Eng., Ankara, Turkey \\ ikara@baskent.edu.tr \\ ${ }^{2}$ Bilkent University, Dept. Ind. Eng., Ankara, Turkey \\ bkara@bilkent.edu.tr \\ ${ }^{3}$ Havelsan A.S., Ankara, Turkey \\ kyetis@yahoo.com
}

\begin{abstract}
This paper proposes a new cost function based on distance and load of the vehicle for the Capacitated Vehicle Routing Problem. The vehicle-routing problem with this new load-based cost objective is called the Energy Minimizing Vehicle Routing Problem (EMVRP). Integer linear programming formulations with $O\left(n^{2}\right)$ binary variables and $O\left(n^{2}\right)$ constraints are developed for the collection and delivery cases, separately. The proposed models are tested and illustrated by classical Capacitated Vehicle Routing Problem (CVRP) instances from the literature using CPLEX 8.0.
\end{abstract}

Keywords: Capacitated vehicle routing problem, Energy minimizing vehicle routing problem, Integer programming.

\section{Introduction}

One of the most important and widely studied combinatorial problem is the Travelling Salesman Problem (TSP) and its variants, which is NP-hard. The problems of finding optimal routes for vehicles from one or several depots to a set of locations/customers are the variants of the multiple Travelling Salesman Problem (m-TSP) and known as Vehicle Routing Problems (VRPs). Vehicle routing problems have many practical applications, especially in transportation and distribution logistics. An extensive literature exists on these problems and their variations (e.g. Golden and Assad 8], Bodin 4, Laporte [12, Laporte and Osman [13, Ball et al. [2], Toth and Vigo [16] [17]).

The Capacitated Vehicle Routing Problem (CVRP) is defined on a graph $G=$ $(V, A)$ where $V=\{0,1,2, \ldots, n\}$ is the set of nodes (vertices), 0 is the depot (origin, home city), and the remaining nodes are customers. The set $A=\{(i, j)$ : $i, j \in V, i \neq j\}$ is an arc (or edge) set. Each customer $i \in V \backslash\{0\}$ is associated with a positive integer demand $q_{i}$ and each $\operatorname{arc}(i, j)$ is associated a travel cost $c_{i j}$ (which may be symmetric, asymmetric, deterministic, random, etc.). There are $m$ vehicles with identical capacity $Q$. The CVRP consists of determining a set of $m$ vehicle routes with minimum cost in such a way that; each route starts and ends at the depot, each customer is visited by exactly one route, and the total demand of each route does not exceed the vehicle capacity $Q$. 
CVRP was first defined by Dantzig and Ramser in 1959 [5]. In that study, the authors used distance as a surrogate for the cost function. Since then, the cost of traveling from node $i$ to node $j$, i.e., $c_{i j}$, has usually been taken as the distance between those nodes (for recent publications, see e.g. Baldacci et al. 1], Letchford and Salazar-Gonzalez [14, Yaman [21]).

The real cost of a vehicle traveling between two nodes depends on many variables: the load of the vehicle, fuel consumption per mile (kilometer), fuel price, time spent or distance traveled up to a given node, depreciation of the tires and the vehicle, maintenance, driver wages, time spent in visiting all customers, total distance traveled, etc. (Baldacci et al.[1], Toth and Vigo [17], Desrochers et al. 6]). Most of the attributes are actually distance or time based and can be approximated by the distance. However, some variables either cannot be represented by the distance between nodes or involve travel costs that may not be taken as constant. Examples of such variables are vehicle load, fuel consumption per mile (kilometer), fuel price or time spent up to a given node. Most of these types of variables may be represented as a function of the flow, especially, as a function of the load of vehicles on the corresponding arc. Thus, for some cases, in addition to the distance traveled, we need to include the load of the vehicle as additional indicator of the cost.

We observe that, some researches with different objectives have been conducted on TSP ( see e.g. Bianco [3], Gouveia and VoB [10, Lucena [15, Tsitsiklis [19). To the best of our knowledge, the vehicle routing literature dealing with single criteria optimization has not previously included the flow on the arcs to the traveling cost, which is the main motivation of this research. In this study, we propose a new cost function which is a product of the distance traveled and the weight of the vehicle on that arc. The contributions of this paper may be summarized as:

- Define a new cost function for vehicle routing problems as a multiple of length of the arc traveled and the total load of the vehicle on this arc. Name this problem as Energy Minimizing Vehicle Routing Problem (EMVRP).

- Present polynomial size integer programming formulations for EMVRP for collection and delivery cases.

We briefly show the relation between the energy used and load of a vehicle and define the new cost function in Section 2. Problem identification and integer programming formulations of the EMVRP for both collection and delivery cases are presented in Section 3. The proposed models are tested and illustrated by standard CVRP instances obtained from the literature and the results are given in Section 4. Concluding remarks are in Section 5.

\section{New Cost Function}

For vehicle routing problems where vehicles carry goods from an origin (center, factory and/or warehouse) to the customer, or from the customer to the origin, the traveling cost between two nodes can be written as,

$$
\text { Cost }=f(\text { load }, \text { distance traveled, others })
$$


where $f($.$) is any function. We derive a cost function that mainly focuses on the$ total energy consumption of the vehicles. Recall from mechanics that,

$$
\text { Work }=\text { force } * \text { distance }
$$

In the CVRP, the movement of the vehicles can be considered as an impending motion where the force causing the movement is equal to the friction force (see for example Walker [20]). Remember also that,

Friction force $=$ Coefficient of friction $*$ weight .

Thus, we have

$$
\begin{aligned}
\text { Work } & =\text { Friction force } * \text { distance } \\
\text { Work } & =\text { Coefficient of friction } * \text { weight } * \text { distance }
\end{aligned}
$$

The coefficient of friction can be considered as constant on roads of the same type. Then, the work done by a vehicle over a link $(i, j)$ will be:

$$
\text { Work }=\text { weight of the vehicle }(\text { over } \operatorname{link}(i, j)) * \operatorname{distance}(\text { of } \operatorname{link}(i, j)) \text {. }
$$

Since work is energy, minimizing the total work done is equivalent to minimizing the total energy used (at least in terms of fuel consumption). Obviously, the weight of the vehicle equals the weight of the empty vehicle (tare) plus the load of the vehicle. Thus, if one wants to minimize the work done by each vehicle, or to minimize the energy used, one needs to use the cost as,

$$
\text { Cost of }(i, j)=[\text { Load of the vehicle over }(i, j)+\text { Tare }] * \text { distance of }(i, j) \text {, }
$$

There seems to be no such definition and objective cost function in the vehicle routing literature. However, there are references on the Internet as shown in Figure 1. (Goodyear website, 22]) indicating that fuel consumption changes with vehicle load.

Figure 1 shows that, miles per gallon decrease with increased vehicle weight. Thus for a CVRP in which goods are carried and fuel prices are relatively more important than the drivers wages, considering the load of the vehicle as well as the distances will produce a more realistic cost of traveling from one customer to another. This analysis shows that for such CVRPs we may define a more realistic cost of traveling from one customer to another by considering the load of the vehicle as well as the distances. We refer the CVRP in which cost is defined as in expression (1) the Energy Minimizing Vehicle Routing Problem (EMVRP).

In the CVRP, vehicles collect and/or deliver the items and/or goods from/to each customer on the route. Thus, the load of a vehicle changes throughout the tour. They show an increasing step function in the case of collection and a decreasing step function in the case of delivery. Thus, load of a vehicle cumulate or accumulate along the tour. For this reason, one must consider the collection and delivery situations, carefully. 


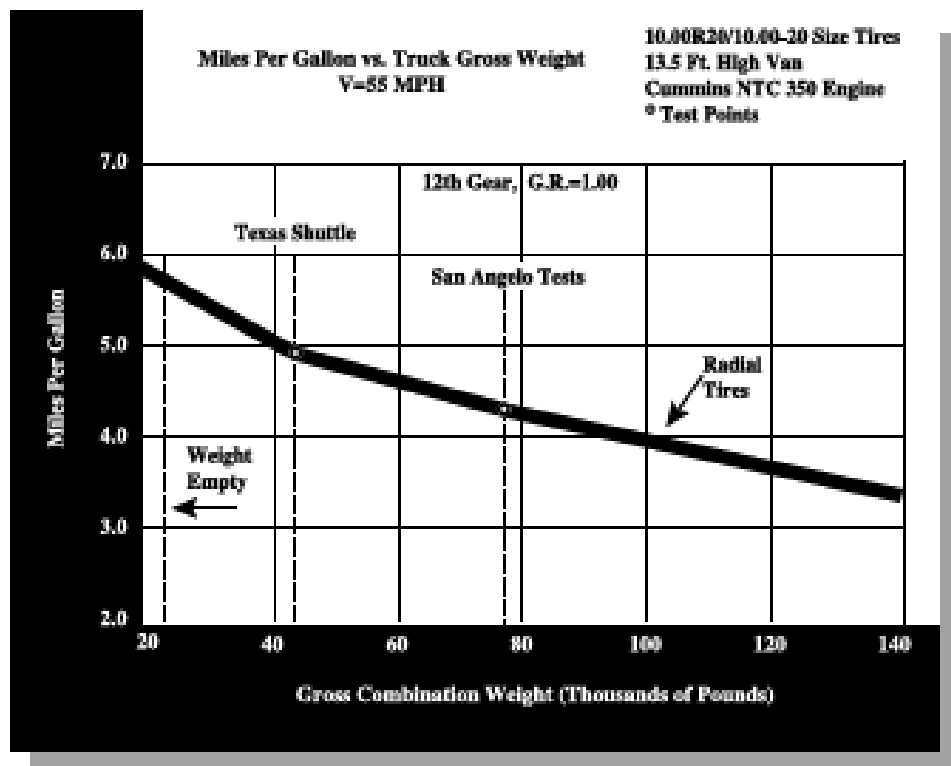

Source, Coodyear Testing Data

Fig. 1. Miles per Gallon versus vehicle weight 22

\section{Integer Programming Formulations}

\subsection{Problem Identification}

Consider a vehicle routing problem defined over a network $G=(V, A)$ where $V=\{0,1,2, \ldots, n\}$ is the node set, 0 is the depot and $A=\{(i, j): i, j \in V, i \neq j\}$ is the set of arcs, and, components of which are given as:

$d_{i j}$ is the distance from node $i$ to node $j$,

$q_{i}$ is the nonnegative weight (e.g. demand or supply) of node $i$,

$m$ is the number of identical vehicles,

$Q_{0}$ is the tare of a vehicle (truck),

$Q$ is the capacity of a vehicle.

We define Energy Minimizing Vehicle Routing Problem (EMVRP) as the problem of constructing vehicle routes such that:

- Each node is served exactly one vehicle,

- Each route starts and ends at the depot,

- The load on the arcs cumulate as much as preceding nodes supply in the case of collection or accumulate as much as preceding nodes demand in the case of delivery,

- The load of a vehicle does not exceed its capacity $Q$,

- The objective is to find a set of $m$ vehicle routes of minimum total cost, i.e., minimum total energy. 
We use the following decision variables in formulating this problem:

$x_{i j}=1$ if the $\operatorname{arc}(i, j)$ is on the tour, and zero otherwise;

$y_{i j}$ is the weight of a vehicle if it goes from $i$ to $j$, and zero otherwise.

Definition of the $y_{i j}$ is the core of this approach. The weight on the first arc of any tour must take a predetermined value, i.e., tare and then must always increase (or decrease) by $q_{i}$ units just after node $i$. In the case of collection, the flow variable shows an increasing step function; for delivery, it shows a decreasing step function. Therefore a model constructed for one case may not be suitable for the other case. The following observation states very important relationship between these situations.

Observation 1. When the distance matrix is symmetric, the optimal route of the delivery (collection) case equals the optimal route of the collection (delivery) case traversed in the reverse order.

Proof. Consider a route which consist of $k$ nodes: $n_{0}-n_{1}-n_{2}-\ldots-n_{k}-n_{0}$, where $n_{0}$ is the depot. For the collection case, the cost of this tour (i.e., total energy used) is:

$$
Q_{0} d_{01}+\sum_{j=1}^{k-1}\left(Q_{0}+\sum_{i=1}^{j} q_{i}\right) d_{j, j+1}+\left(Q_{0}+\sum_{i=1}^{k} q_{i}\right) d_{k 0}
$$

For the delivery case, the cost of the reverse route $n_{0}-n_{k}-n_{k-1}-\ldots-n_{1}-n_{0}$ is:

$$
\left(Q_{0}+\sum_{i=1}^{k} q_{i}\right) d_{0 k}+\sum_{j=1}^{k-1}\left(Q_{0}+\sum_{i=1}^{j} q_{i}\right) d_{j+1, j}+Q_{0} d_{10}
$$

Observe that (2) and (3) are the same for symmetric $D=[d i j]$ matrices.

\subsection{Formulations}

For the symmetric-distance case, one does not need to differentiate between collection and delivery since the solution of one will determine the solution of the other. For the case of an asymmetric distance matrix, due to the structure of the problem, we present decision models for collection and delivery cases, separetely. The model for the collection case is:

$$
F_{1}: \operatorname{Min} \sum_{i=0}^{n} \sum_{j=0}^{n} d_{i j} y_{i j}
$$




$$
\begin{aligned}
& \text { s.t. } \\
& \sum_{i=1}^{n} x_{0 i}=m \\
& \sum_{i=1}^{n} x_{i 0}=m \\
& \sum_{i=0}^{n} x_{i j}=1, \quad j=1,2, \ldots, n \\
& \sum_{j=0}^{n} x_{i j}=1, \quad i=1,2, \ldots, n \\
& \sum_{\substack{j=0 \\
j \neq i}}^{n} y_{i j}-\sum_{\substack{j=0 \\
j \neq i}}^{n} y_{j i}=q_{i}, \quad i=1,2, \ldots, n \\
& y_{0 i}=Q_{0} x_{0 i}, \quad i=1,2, \ldots, n \\
& y_{i j} \leq\left(Q+Q_{0}-q_{j}\right) x_{i j}, \quad(i, j) \in A \\
& y_{i j} \geq\left(Q_{0}+q_{i}\right) x_{i j}, \quad \forall(i, j) \in A \\
& x_{i j}=0 \text { or } 1, \quad(i, j) \in A
\end{aligned}
$$

where $q_{0}=0$.

The cost of traversing an arc $(i, j)$ is the product of the distance between the nodes $i$ and $j$ and weight on this arc and this is satisfied by the objective function given in (4). Constraints (5) and (6) ensure that $m$ vehicles are used. Constraints (7) and (8) are the degree constraints for each node. Constraint (9) is the classical conservation of flow equation balancing inflow and outflow of each node, which also prohibits any illegal subtours. Constraint (10) initialize the flow on the first arc of each route, cost structure of the problem necessitates such an initialization. Constraints (11) take care of the capacity restrictions and forces $y_{i j}$ to zero when the arc $(i, j)$ is not on any route, and constraint (12) produce lower bounds for the flow on any arc. Integrality constraints are given in (13). We do not need nonnegativity constraints since we have constraints given in (12). Let us call constraints (10), (11) and (12) as the bounding constraints of the formulation. Validity of them is shown in proposition 1 below.

Proposition 1. In the case of collection, the constraints given in (10), (11) and (12) are valid for EMVRP.

Proof. As it is explained before, we need initialization value of $y_{i j}$ s for each tour that constraints $(10)$ do it, otherwise $y_{i j}$ s may not be the actual weight on the arcs. Constraints (12) is valid since going from $i$ to $j$ the flow must be at least the initial value plus the weight of the node $i$ (unless node $i$ is the depot, in which case $\left.q_{0}=0\right)$. Similarly, since the vehicle is destined for node $j$, it will also collect the $q_{j}$ units at node $j$ (unless $j$ is the depot). In that case, the flow on the arc upon arriving at node $j$ should be enough to take the weight of node 
$j\left(q_{j}\right)$, i.e., $y_{i j}+q_{j} x_{i j} \leq\left(Q+Q_{0}\right) x_{i j}$, which produce constraints (11). Similar constraints for classical CVRP may be seen in (Gouveia [9], Baldacci et al. 1], Letchford and Salazar-Gonzalez [14, Yaman [21]).

Due to Observation 1, the delivery problem for the symmetric case need not be discussed. For the asymmetric case, the delivery problem will be modeled by replacing constraints (9),(10), (11) and (12) with the following given below.

$$
\begin{aligned}
\sum_{\substack{j=0 \\
j \neq i}}^{n} y_{i j}-\sum_{\substack{j=0 \\
j \neq i}}^{n} y_{j i}=q_{i}, & i=1,2, \ldots, n \\
y_{i 0}=Q_{0} x_{i 0}, & i=1,2, \ldots, n \\
y_{i j} \leq\left(Q+Q_{0}-q_{i}\right) x_{i j}, & \forall(i, j) \in A \\
y_{i j} \geq\left(Q_{0}+q_{j}\right) x_{i j}, & \forall(i, j) \in A
\end{aligned}
$$

Thus the model for the delivery case is:

$$
\begin{aligned}
& F_{2}: \operatorname{Min} \sum_{i=0}^{n} \sum_{j=0}^{n} d_{i j} y_{i j} \\
& \text { s.t. (5)-(8),(13) - (17). }
\end{aligned}
$$

where $q_{0}=0$.

Both of the proposed models have $n^{2}+n$ binary and $n^{2}+n$ continuous variables, and $2 n^{2}+6 n+2$ constraints, thus proposed formulations contain $O\left(n^{2}\right)$ binary variables and $O\left(n^{2}\right)$ constraints.

\subsection{Extension to Distance Constraints}

In certain applications of the CVRP, there is an additional restriction on the total distance traveled by each vehicle (or cost, or time). This problem is known as the Distance-Constrained VRP (abbreviated as DVRP). In the case of the EMVRP, if such a side condition is imposed, we may easily put the necessary constraints into the proposed models. We need to define additional decision variables as:

$z_{i j}$ the total distance traveled by a vehicle (or cost, or time) from the origin to node $j$ when it goes from $i$ to $j$.

Note that if the $\operatorname{arc}(i, j)$ is not on the optimal route, then $z_{i j}$ must be equal to zero. The distance-constrained EMVRP can be modeled by including constraints (18)-(21) in both collection and delivery cases.

$$
\begin{array}{rl}
\sum_{\substack{j=0 \\
j \neq i}}^{n} z_{i j}-\sum_{\substack{j=0 \\
j \neq i}}^{n} z_{j i}=\sum_{j=0}^{n} d_{i j} x_{i j} & i=1,2, \ldots, n \\
z_{i j} \leq\left(T-d_{j 0}\right) x_{i j} & j \neq 0,(i, j) \in A \\
z_{0 i}=d_{0 i} x_{0 i} & i=1,2, \ldots, n \\
z_{i j} \geq\left(d_{0 i}+d_{i j}\right) x_{i j} & i \neq 0(i, j) \in A
\end{array}
$$


where $T$ is the maximum distance that a vehicle can travel. These constraints are taken from Kara [11. Constraints (18) sum the value of the $z_{i j}$ s and eliminate all illegal subtours. Constraints (19), (20)and (21) are the distance bounding constraints ensuring that the total distance of each route cannot exceed the predetermined value $T$.

\section{Illustrative Examples}

In this section, we conduct some numerical examples of EMVRP formulation focusing on the collection case. We use two CVRP instances from the literature and we solve the instances via CPLEX 8.0 on an Intel Pentium III $1400 \mathrm{MHz}$ computer. We want to test the effect of the new objective function on the optimal routes (i.e. distance-based routes versus energy-based routes). For that purpose we define two scenarios. Scenario 1 is the EMVRP and Scenario 2 is the standard CVRP (distance minimizing CVRP, which tries to minimize the total distances without considering the vehicle loads). We choose 2 symmetric instances, eil3 and gr17 from the literature 23. For eil3 $m=4$ and $Q=6000$, and for gr-17 $m=3$ and $Q=6$. For each problem, we assume $Q_{0}=15 \%$ of $Q$. Table 1 summarizes the results. The second and third columns of Table 1 provide the solutions of Scenario 1 and 2 of eil 3 and the $4^{\text {th }}$ and $5^{\text {th }}$ columns provide those of gr-17.

Table 1. Computational Results for eil3 and gr-17 Problems [23]

\begin{tabular}{|l|c|c|c|c|}
\hline & \multicolumn{2}{|c|}{ eil3 } & \multicolumn{2}{c|}{ gr-17 } \\
\cline { 2 - 5 } & $\begin{array}{c}\text { Scenario 1 } \\
\text { EMVRP }\end{array}$ & $\begin{array}{c}\text { Scenario 2 } \\
\text { CVRP }\end{array}$ & $\begin{array}{c}\text { Scenario 1 } \\
\text { EMVRP }\end{array}$ & $\begin{array}{c}\text { Scenario 2 } \\
\text { CVRP }\end{array}$ \\
\hline Energy Min. & 779.400 & 810.700 & 7331 & 8810 \\
\hline Distance Min. & 277 & 247 & 3088 & 2685 \\
\hline \multirow{3}{*}{ Selected } & $0-4-7-10-6-0$ & $0-1-0$ & $0-1-4-10-2-5-16-0$ & $0-12-16-13-5-7-6-0$ \\
Routes & $0-8-5-2-0$ & $0-8-5-3-0$ & $0-9-14-13-7-6-0$ & $0-14-9-1-4-10-2-0$ \\
& $0-9-12-0$ & $0-9-12-10-6-0$ & $0-15-11-8-3-12-0$ & $0-15-11-8-3-0$ \\
\hline
\end{tabular}

As Table 1 demonstrates, there is a considerable difference between energyminimizing and distance-minimizing solutions. The cost of the route that minimizes total distance may be $13 \%$ less than the solution which minimizes energy. Counter intuitively for both examples, energy usage increases as total distance decreases. Observe from Table 1 that the routes selected under two scenarios are completely different.

Even though we propose a model with $O\left(n^{2}\right)$ binary variables and $O\left(n^{2}\right)$ constraints for the EMVRP, the CPU times of CPLEX over moderate sized problems were not promising. It is therefore necessary to develop efficient solution 
procedures for the EMVRP like heuristics proposed for CVRP (Gendreau, et al. [7, Toth and Vigo [18]). However, these modifications are beyond the scope of this paper.

\section{Conclusion}

This paper proposes a new objective function for the vehicle routing problem in which goods are carried and fuel prices are relatively more important than the drivers wages. For such CVRPs we define a more realistic cost of traveling from one customer to another by considering the load of the vehicle as well as the distances. We refer the CVRP as the Energy Minimizing Vehicle Routing Problem (EMVRP), where cost is defined as a multiple of length of the arc traveled and the total load of the vehicle on this arc.

Integer programming formulations with $O\left(n^{2}\right)$ binary variables and $O\left(n^{2}\right)$ constraints are developed for both collection and delivery cases of EMVRP. The adaptability of the formulations to the distance-constrained case is also demonstrated. The proposed models are tested and demonstrated by using CPLEX 8.0 on two instances taken from the literature.

The adaptability and usability of the proposed models to the other network design problems, such as multiple traveling repairman problem and school-bus routing problems, are under consideration.

\section{References}

1. Baldacci, R., Hadjiconstantinou, E., Mingozzi, A.: An exact algorithm for the capacitated vehicle routing problem based on a two-commodity network flow formulation. Operations Research 52, 723-738 (2004)

2. Ball, M.O., Magnanti, T.L., Monma, C.L., Nemhauser, G.L.: Handbooks in Operations Research and Management Science: Network routing. North-Holland, Amsterdam (1995)

3. Bianco, L., Mingozzi, A., Ricciardelli, S.: The traveling salesman problem with cumulative costs. Networks 23, 81-91 (1993)

4. Bodin, L.D.: Twenty years of routing and scheduling. Operations Research 38(4), 571-579 (1990)

5. Dantzig, G.B., Ramser, J.H.: The truck dispatching problem. Management Science 6, 80-91 (1959)

6. Desrochers, M., Lenstra, J.K., Savelsbergh, M.W.P.: A classification scheme for vehicle routing and scheduling problems. European Journal of Operational Research 46, 322-332 (1990)

7. Gendreau, M., Hertz, A., Laporte, G.: A tabu search heuristic for the vehicle routing problem. Management Science 40, 1276-1290 (1994)

8. Golden, B.L., Assad, A.A.: Vehicle Routing: Methods and Studies. North-Holland, Amsterdam (1988)

9. Gouveia, L.: A result on projection for the vehicle routing problem. European Journal of Operational Research 85, 610-624 (1995)

10. Gouveia, L., VoB, S.: A classification of formulations for the (time-dependent) traveling salesman problem. European Journal of Operational Research 83, 69-82 (1995) 
11. Kara, İ.: Flow based Integer programming formulations for the distance constrained vehicle routing problem. Technical report 2006/01, Başkent University Faculty of Engineering, Ankara, Turkey (2006)

12. Laporte, G.: The vehicle routing problem: An overview of exact and approximate algorithms. European Journal of Operational Research 59, 345-358 (1992)

13. Laporte, G., Osman, I.H.: Routing problems: A bibliography. Annals of Operations Research 61, 227-262 (1995)

14. Letchford, A.N., Salazar-Gonzalez, J-J.: Projection results for vehicle routing. Mathematical Programming, Ser. B 105, 251-274 (2006)

15. Lucena, A.: Time-dependent traveling salesman problem-The deliveryman case. Networks 20, 753-763 (1990)

16. Toth, P., Vigo, D. (eds.): The Vehicle Routing Problem. SIAM Monographs on Discrete Mathematics and Applications. SIAM (2002)

17. Toth, P., Vigo, D.: An overview of vehicle routing problems. In: Toth, P., Vigo, D. (eds.) The Vehicle Routing Problem. SIAM Monographs on Discrete Mathematics and Applications, pp. 1-26. SIAM (2002)

18. Toth, P., Vigo, D.: The Granular Tabu Search and its application to the vehicle routing problem. INFORMS Journal on Computing 15(4), 333-346 (2003)

19. Tsitsiklis, J.N.: Special cases of traveling salesman and repairman problems with time windows. Networks 22, 263-282 (1992)

20. Walker, K.M.: Applied Mechanics for Engineering Technology, 6th edn. Prentice Hall, Englewood Cliffs (2000)

21. Yaman, H.: Formulations and valid inequalities for the heterogeneous vehicle routing problem. Mathematical Programming, Ser. A 106, 365-390 (2006)

22. Goodyear web site:

http://www.goodyear.com/truck/pdf/commercialtiresystems/FuelEcon.pdf

23. VRPLIB:

http://www.or.deis.unibo.it/research_pages/ORinstances/VRPLIB/

VRPLIB.html 